\title{
Towards Designing Benchmarks for Humanoid Space Robots: Northeastern's NASA Valkyrie Dataset
}

\author{
Velin Dimitrov ${ }^{1}$, Murphy Wonsick ${ }^{1}$, Xianchao Long $^{1}$, Pauline Maurice ${ }^{1}$, Dagmar Sternad ${ }^{1}$, \\ and Taskin Padir ${ }^{1}$ \\ ${ }^{1}$ Northeastern University
}

May 5, 2020

\begin{abstract}
Designing benchmarks for algorithms developed to run on physical robot hardware remains to be a challenge. Towards achieving benchmarks for space humanoid robots, we present Northeastern's humanoid robot dataset containing physical sensor data from NASA's Valkyrie (R5), including robot pose estimate, joint angles and velocities, center of pressure, center of mass, ground reaction wrenches, and motion capture ground truth pose. The dataset includes various mobility and manipulation tasks as atomic robot behaviors including walking and reaching motions. Inspired by the NASA Space Robotics Challenge, the dataset is intended for use by the community that wishes to conduct humanoid robot research without direct access to a hardware platform. In addition, it will enable comparative studies in terms of hardware designs, as well as task and motion planning methods. The dataset will provide the humanoid robotics research community with a resource not only to bridge the gap between simulation-based and experimental algorithm validation but also to design task-level benchmarks for humanoid space robots. This paper describes the robot hardware, software, data collection process, post-processing steps, and structure of data for Northeastern's NASA Valkyrie dataset.
\end{abstract}

\section{Hosted file}

main.pdf available at https://authorea.com/users/298645/articles/427981-towards-designingbenchmarks-for-humanoid-space-robots-northeastern-s-nasa-valkyrie-dataset 